\title{
EDITORIAL
}

\section{Narrative reviews from a fraudulent author: reasons to retract}

\author{
Christiane S. Hartog ${ }^{1,2^{*}}$ (D) and Anders Perner ${ }^{3}$
}

(0) 2019 Springer-Verlag GmbH Germany, part of Springer Nature

Clinical studies from fraudulent authors are retracted, but narrative reviews are not. We believe that reviews should be considered for retraction, too. J. Boldt is number 2 on the top list of fraudulent authors; currently, 96 of his clinical studies have been retracted since 2010. Earlier trials from the 1990s are also suspected for fraudulence, but the ethics boards no longer keep the documents, making it difficult to prove [1]. Fraudulent clinical studies generate false evidence that may harm patients $[1,2]$. Narrative reviews based on falsified data generate false messages that may also result in patient harm. The effect of narrative reviews on clinical practise should not be underestimated. We believe that narrative reviews may be quite influential especially in contentious fields. Readers may prefer narrative reviews because they are easy to follow and contain clear recommendations [3], while systematic reviews, though considered to be of higher quality, may appear "boring and sometimes unimplementable" [4] and contain no or limited recommendations. Indeed, leading journals publish more narrative than systematic reviews [5].

Boldt published numerous narrative reviews. We previously found that he alone authored 21 of all 124 reviews supporting the use of hydroxyethyl starch (HES) published between 1975 and 2010 [6]. From the 1990s onwards, favourable reviews increased from two to eight per year and HES's share of the artificial colloid market tripled from 20 to $60 \%$ [6]. We believe this association implies that narrative reviews contributed to the increasing use of HES. Table 1 shows an overview over 23 reviews written between 1998 and 2010 (21 previously

*Correspondence: Christiane.hartog@mailbox.org

1 Department of Anesthesiology and Intensive Care Medicine, Charité Universitätsmedizin Berlin, Charitéplatz 1, 10117 Berlin, Germany

Full author information is available at the end of the article identified reviews [6] plus 2 reviews identified during an update in November 2018). Closer scrutiny reveals that all reviews cite retracted studies and contain misleading statements supporting the use of a substance that is less effective and clearly harmful in some patient groups. The misleading messages (Table 1) can also be found in reviews written by leading medical officers from a manufacturing company $[7,8]$. Boldt admitted to financial payments from the fluid manufacturer to himself and his department [9]. However, the sheer multitude of reviews is astounding (not counting the numerous clinical studies by Boldt) as is the fact that several reviews contain the results from different MEDLINE searches, complex graphs and reference lists with more than 100 references. It may therefore be questioned whether Boldt wrote all these reviews himself.

The false claims have been refuted by the results of clinical trials and meta-analyses [10-13]. The EMA (European Medicines Agency) has issued clear constraints for using HES in critically ill patients, including new measures introduced this year to protect patients further (https://www.ema.europa.eu/en/medicines/human/ referrals/hydroxyethyl-starch-hes-containing-medicinalproducts). However, HES is used widely outside Europe, and Boldt's narrative reviews continue to be cited. Web of Science Citation reports show 591 citations overall and 238 since 2011. In his reviews, Boldt bases his misleading claims on his own fraudulent studies (Table 1). Only one review was withdrawn as it contained Boldt's retracted contribution to German guidelines on blood product use [14]. Not surprisingly, Boldt had deprecated albumin in favour of starches.

Narrative reviews relying on falsified data should therefore be retracted too. 
Table 1 Overview over 23 narrative reviews of HES by J. Boldt

\begin{tabular}{|c|c|c|c|c|c|c|c|}
\hline \multirow{2}{*}{$\begin{array}{l}\text { Refer- } \\
\text { ences } \\
\text { no. }\end{array}$} & \multirow[t]{2}{*}{ Year } & \multicolumn{2}{|c|}{ Times cited } & \multirow[t]{2}{*}{ Focus } & \multicolumn{2}{|c|}{ Reference to own studies } & \multirow[t]{2}{*}{ Misleading messages and unsubstantiated claims } \\
\hline & & All & 2011-18 & & Re-tracted & Not re-tracted & \\
\hline 1 & 1998 & n.a. & n.a. & ICU & 2 & 6 & $\begin{array}{l}\text { Deprecates albumin } \\
\text { Denies HES class effect, i.e.,"modern" HES is less harmful and } \\
\text { has more benefits than "older" HES preparations } \\
\text { Claims "modern" HES is safer } \\
\text { Claims additional beneficial effects of "modern" HES (microcir- } \\
\text { culation, inflammation, coagulation) } \\
\text { Claims Crys-coll ratio } 3 \text { or } 4 \text { to } 1\end{array}$ \\
\hline 2 & 1999 & 8 & 2 & Surgery & 2 & 2 & $\begin{array}{l}\text { Claims colloid more effective } \\
\text { Deprecates albumin } \\
\text { Claims HES more effective and safer than other synthetic } \\
\text { colloids } \\
\text { Denies HES class effect } \\
\text { Claims additional beneficial effects }\end{array}$ \\
\hline 3 & 1999 & 11 & 1 & Cardiac surgery & 0 & 10 & $\begin{array}{l}\text { Deprecates deprecates crystalloids } \\
\text { Denies HES class effect } \\
\text { Claims "modern" HES has no harmful effects on coagulation }\end{array}$ \\
\hline 4 & 2000 & 28 & 7 & Surgery & 1 & 3 & $\begin{array}{l}\text { Deprecates crystalloids } \\
\text { Deprecates albumin } \\
\text { Denies HES class effect } \\
\text { Claims additional beneficial effects }\end{array}$ \\
\hline 5 & 2000 & 22 & 4 & ICU & 1 & 2 & $\begin{array}{l}\text { Deprecates albumin } \\
\text { Denies HES class effect } \\
\text { Denies mortality is important outcome in fluid trials } \\
\text { Claims additional beneficial effects }\end{array}$ \\
\hline 6 & 2002 & 12 & 1 & Renal failure & 2 & 1 & Claims "modern" HES is not nephrotoxic \\
\hline 7 & 2003 & 37 & 7 & Renal failure & 1 & 2 & $\begin{array}{l}\text { Claims "modern" HES is not nephrotoxic } \\
\text { Claims adequate hydration can protect from nephrotoxic HES } \\
\text { effects }\end{array}$ \\
\hline 8 & 2003 & 53 & 8 & General & 5 & 3 & $\begin{array}{l}\text { Deprecates albumin } \\
\text { Denies HES class effect } \\
\text { Claims additional beneficial effects; claims safety and efficacy } \\
\text { of modern HES is proven by numerous studies }\end{array}$ \\
\hline 9 & 2003 & 20 & 2 & Renal failure & 1 & 2 & $\begin{array}{l}\text { Denies HES class effect } \\
\text { Claims "modern" HES is not nephrotoxic } \\
\text { Claims adequate hydration can protect from nephrotoxic HES } \\
\text { effects }\end{array}$ \\
\hline 10 & 2004 & 7 & 1 & General & 4 & 4 & $\begin{array}{l}\text { Deprecates albumin } \\
\text { Denies HES class effect } \\
\text { Claims additional beneficial effects; claims spurious new } \\
\text { research questions }\end{array}$ \\
\hline 11 & 2004 & 52 & 9 & Trauma & 4 & 3 & $\begin{array}{l}\text { Claims that colloids are more effective for resuscitation } \\
\text { Deprecates albumin } \\
12 \text { Claims that better HES are on the market in Europe than in } \\
\text { North America }\end{array}$ \\
\hline 12 & 2005 & 50 & 17 & General & 3 & 0 & $\begin{array}{l}\text { Deprecates albumin } \\
\text { Denies mortality is important outcome in fluid trials } \\
\text { Claims additional beneficial effects by direct action of the HES } \\
\text { molecule }\end{array}$ \\
\hline 13 & 2005 & 16 & 1 & General & 2 & 0 & $\begin{array}{l}\text { Optimum colloid should be defined by physiological criteria, } \\
\text { not by mortality in clinical trials }\end{array}$ \\
\hline 14 & 2006 & 14 & 3 & Cardiac surgery & 3 & 1 & $\begin{array}{l}\text { Deprecates US meta-analyses because they ignore claimed } \\
\text { additional beneficial effects of HES } \\
\text { Claims safety and efficacy of modern HES is proven by numer- } \\
\text { ous studies }\end{array}$ \\
\hline 15 & 2006 & 33 & 12 & General & 4 & 3 & $\begin{array}{l}\text { Deprecates crystalloids } \\
\text { Deprecates albumin } \\
\text { Claims additional beneficial effects of the HES molecule }\end{array}$ \\
\hline 16 & 2006 & 30 & 12 & Abdominal surgery & 3 & 0 & $\begin{array}{l}\text { Deprecates crystalloids } \\
\text { Claims additional beneficial effects of the HES molecule }\end{array}$ \\
\hline
\end{tabular}


Table 1 (continued)

\begin{tabular}{|c|c|c|c|c|c|c|c|}
\hline \multirow{2}{*}{$\begin{array}{l}\text { Refer- } \\
\text { ences } \\
\text { no. }\end{array}$} & \multirow[t]{2}{*}{ Year } & \multicolumn{2}{|c|}{ Times cited } & \multirow[t]{2}{*}{ Focus } & \multicolumn{2}{|c|}{ Reference to own studies } & \multirow[t]{2}{*}{ Misleading messages and unsubstantiated claims } \\
\hline & & All & 2011-18 & & Re-tracted & Not re-tracted & \\
\hline 17 & 2007 & 10 & 1 & Cardiac surgery & 3 & 0 & $\begin{array}{l}\text { Deprecates albumin } \\
\text { Claims modern HES is not harmful to coagulation } \\
\text { Claims additional beneficial effects of the HES molecule }\end{array}$ \\
\hline 18 & 2008 & 16 & 6 & Balanced HES solutions & 3 & 1 & Claims additional benefits through balanced carrier solution \\
\hline 19 & 2009 & 23 & 11 & ICU, renal failure & 3 & 0 & $\begin{array}{l}\text { Claims modern HES is not nephrotoxic } \\
\text { Nephrotoxic effects can be avoided by proper hydration }\end{array}$ \\
\hline 20 & 2009 & 43 & 33 & General & 7 & 0 & $\begin{array}{l}\text { Denies HES class effects } \\
\text { Claims additional beneficial effects of the HES molecule } \\
\text { Claims numerous studies have proven safety and efficacy of } \\
\text { "modern" HES } \\
\text { Claims mortality is not influenced by choice of fluid }\end{array}$ \\
\hline 21 & 2010 & 6 & 4 & General & 2 & 0 & Claims spurious new research problems \\
\hline 22 & 2010 & 37 & 37 & $\begin{array}{l}\text { Microcirculation and } \\
\text { tissue oxygenation }\end{array}$ & 1 & 0 & Suggests additional beneficial effects of the HES molecule \\
\hline 23 & 2010 & 63 & 59 & Albumin vs. HES & 2 & 0 & $\begin{array}{l}\text { Deprecates albumin } \\
\text { Claims "modern" HES is equally effective and safe }\end{array}$ \\
\hline
\end{tabular}

References 1-23 are listed in the Online Supplement

\section{Electronic supplementary material}

The online version of this article (https://doi.org/10.1007/s00134-019-05558-5) contains supplementary material, which is available to authorized users.

\section{Author details}

${ }^{1}$ Department of Anesthesiology and Intensive Care Medicine, Charité Universitätsmedizin Berlin, Charitéplatz 1, 10117 Berlin, Germany. ${ }^{2}$ KLINIK BAVARIA Kreischa, An der Wolfsschlucht 1-2, 01731 Kreischa, Germany. ${ }^{3}$ Department of Intensive Care, Rigshospitalet, University of Copenhagen, Copenhagen, Denmark.

\section{Compliance with ethical standards}

\section{Conflicts of interest}

$\mathrm{CSH}$ reports grants from the German Federal Ministry of Education and Research (BMBF) via the Center for Sepsis Control and Care (CSCC; FKZ: 01EO1002) and the Innovations Funds/Federal Joint Committee FKZ 01VSF17010 and ESICM (European Society of Intensive Care Medicine). AP reports that his ICU receives funds for research from Ferring Pharmaceuticals. He holds grants from the Novo Nordisk Foundation and Innovation Foundation Denmark.

\section{Ethical approval}

An approval by an ethics committee was not applicable.

\section{Publisher's Note}

Springer Nature remains neutral with regard to jurisdictional claims in published maps and institutional affiliations.

Received: 10 January 2019 Accepted: 2 February 2019

Published online: 14 February 2019

\section{References}

1. Marcus A (2018) A scientist's fraudulent studies put patients at risk. Science $362: 394$

2. Wiedermann CJ, Joannidis M (2018) The Boldt scandal still in need of action: the example of colloids 10 years after initial suspicion of fraud. Intensive Care Med 44:1735-1737
3. Loke YK, Derry S (2003) Does anybody read "evidence-based" articles? BMC Med Res Methodol 3:14

4. Greenhalgh T (2012) Outside the box: why are cochrane reviews so boring? Br J Gen Pract 62:371

5. Faggion CM Jr, Bakas NP, Wasiak J (2017) A survey of prevalence of narrative and systematic reviews in five major medical journals. BMC Med Res Methodol 17:176

6. Hartog CS, Skupin H, Natanson C, Sun J, Reinhart K (2012) Systematic analysis of hydroxyethyl starch (HES) reviews: proliferation of low-quality reviews overwhelms the results of well-performed meta-analyses. Intensive Care Med 38:1258-1271

7. Treib J, Baron JF, Grauer MT, Strauss RG (1999) An international view of hydroxyethyl starches. Intensive Care Med 25:258-268

8. Westphal M, James MF, Kozek-Langenecker S, Stocker R, Guidet B, Van Aken H (2009) Hydroxyethyl starches: different products—different effects. Anesthesiology 111:187-202

9. Boldt J, Ince C (2010) The impact of fluid therapy on microcirculation and tissue oxygenation in hypovolemic patients: a review. Intensive Care Med 36:1299-1308

10. Myburgh JA, Finfer S, Bellomo R, Billot L, Cass A, Gattas D, Glass P, Lipman J, Liu B, McArthur C, McGuinness S, Rajbhandari D, Taylor CB, Webb SA (2012) Hydroxyethyl starch or saline for fluid resuscitation in intensive care. N Engl J Med 367:1901-1911

11. Perner A, Haase N, Guttormsen AB, Tenhunen J, Klemenzson G, Aneman A, Madsen KR, Moller MH, Elkjaer JM, Poulsen LM, Bendtsen A, Winding R, Steensen M, Berezowicz P, Soe-Jensen P, Bestle M, Strand K, Wiis J, White JO, Thornberg KJ, Quist L, Nielsen J, Andersen LH, Holst LB, Thormar K, Kjaeldgaard AL, Fabritius ML, Mondrup F, Pott FC, Moller TP, Winkel P, Wetterslev J (2012) Hydroxyethyl starch 130/0.42 versus Ringer's acetate in severe sepsis. N Engl J Med 367:124-134

12. Hartog CS, Natanson C, Sun J, Klein HG, Reinhart K (2014) Concerns over use of hydroxyethyl starch solutions. BMJ 349:5981

13. Orbegozo Cortes D, Gamarano Barros T, Njimi H, Vincent JL (2015) Crystalloids versus colloids: exploring differences in fluid requirements by systematic review and meta-regression. Anesth Analg 120:389-402

14. Boldt J (2010) Guidelines on therapy with blood components and plasma derivatives: human albumin recommendations of the scientific advisory board of the Medical Council. Der Anaesthesist 59:566-574 